\title{
A INFLUÊNCIA DAS FORÇAS ACELERATIVAS NO TRANSPORTE DE PACIENTES COM AVC HEMORRÁGICO EM AERONAVES DE ASA FIXA
}

Felipe Trevisan Matos NOVAK 1 , Heloisa Giccele Claudino NOVAK², Heitor Pinheiro LIMA NETO ${ }^{3}$ e Michelle TAVERNA ${ }^{4}$

\section{RESUMO}

A doença hemorrágica intracraniana é uma condição grave, que necessita de investigação e tratamento em grandes centros de atenção à saúde. $O$ transporte aeromédico com asa fixa, em muitos casos, é a forma mais rápida de transportar estes pacientes, entretanto os submete a forças acelerativas que podem agravar seu quadro clínico. Este trabalho se trata de uma pesquisa bibliográfica com análise crítica e leitura sistemática de livros, sites e artigos cujo objetivo foi caracterizar a produção científica especializada acerca da influência das forças acelerativas em pacientes com AVC hemorrágico, em bases de dados, publicada através da Medical Literature Analysis and Retrieval System Online (MEDLINE) via Pubmed. Destaca-se escassa inclusão do conteúdo específico e a existência de muitas lacunas na produção de conhecimento sobre o tema. Pesquisas que analisam esta interface qualificam o cuidado no posicionamento do paciente na aeronave; elevação da cabeceira do leito; monitoramento contínuo do paciente; preferência pelo uso de pista longa; redução de manobras bruscas em voo; aceleração e desaceleração da aeronave controladas. Enfatizando a complexidade e a especificidade na remoção dos pacientes neurológicos, se torna evidente a necessidade de atualização e melhores definições de protocolos que evidenciem maior qualidade na assistência prestada nas transferências aéreas de pacientes com AVC hemorrágico.

Palavras-chave: transporte aeromédico, AVC hemorrágico, força G.

\section{INTRODUÇÃO}

Os Acidentes Vasculares Cerebrais hemorrágicos (AVCh) correspondem de 20 - 30\% dos eventos vasculares encefálicos agudos, apresentam grande morbi-mortalidade e acometem diversas faixas etárias. O tratamento deve ser feito em centros especializados, com equipamentos de alta complexidade e profissionais treinados, 0 que implica na necessidade de transporte destes pacientes (CAPLAN, 2009).

O uso de aeronaves de asa fixa (aviões) ou asa rotativa (helicópteros) pode abreviar o tempo de chegada a um centro especializado e permite a redução do tempo de internação (PHILLIPS et al., 2013; NAEMT, 2017), traz benefícios na sobrevivência e reduz morbidade após o evento (SILBERGLEIT et al., 2003). Porém, a realização do

1. Médico de voo Helisul, Membro do Dep. de Neurociências da ABRAERO, Especialista em Transporte e Resgate Aeromédico pela Faculdade Inspirar, Curitiba, Paraná, Brasil. felipenovak@gmail.com

2. Acadêmica de Medicina das Faculdades Pequeno Príncipe, Curitiba, Paraná, Brasil. heloisanovak@hotmail.com

3. Coordenador da pós-graduação de Transporte e Resgate Aeromédico da Faculdade Inspirar, Curitiba, Paraná, Brasil. heitorpinheironeto@terra.com.br

4. Enfermeira de Voo Helisul, 1 Ten da Força Aérea Brasileira, Presidente da ABRAERO, Curitiba, Paraná, Brasil. michelletaverna@hotmail.com 


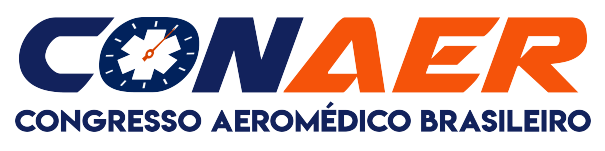

transporte aeromédico com aeronaves de asa fixa está sujeita a influência de forças acelerativas que podem trazer danos clínicos aos pacientes (DAVIS, 2008).

Para elucidar o papel destas forças nos pacientes acometidos pelos AVCh e buscar esclarecer dúvidas sobre a possibilidade da realização ou não destes transportes, realizamos revisão da literatura científica disponível até o momento.

\section{METODOLOGIA}

Trata-se de uma revisão bibliográfica acerca do transporte aeromédico de pacientes acometidos por AVCh, a partir da análise crítica e leitura sistemática de livros, sites e artigos científicos selecionados através de busca na base de dados Medical Literature Analysis and Retrieval System Online (MEDLINE) via Pubmed, utilizando as palavras-chave: hemorrhagic stroke, aerospace medicine, acceleration forces, aircraft e de bases de dados epidemiológicos como DATASUS. Foram excluídos artigos relacionados ao transporte de pacientes com a utilização de aeronaves de asa rotativa. O período de estudo foi de 1972 a 2021, visto que na neurociência alguns clássicos literários reverberam até os dias atuais.

\section{RESULTADOS E DISCUSSÕES}

As doenças do sistema circulatório são as que mais acometem pacientes adultos a partir dos 40 anos no Brasil. Dentre estas doenças, os Acidentes Vasculares Cerebrais (AVC) respondem pela terceira maior causa de mortalidade no Brasil cerca de 52,4 óbitos/100.000 habitantes segundo os Indicadores de Dados Básicos de 2012 (IDB, 2012). Do total de AVCs, cerca de 20-30\% ocorrem por hemorragia intracraniana - AVCh (Caplan, 1988). Parte destas pode aumentar nas primeiras 24-48 horas devido à persistência da pressão arterial elevada ou do sangramento no leito inicial, edema perilesional ou lesão de microcirculação (CAPLAN, 2009).

O tratamento do AVCh consiste nas medidas básicas e avançadas de suporte à vida e o tratamento específico da hemorragia e da sua causa. As medidas iniciais e avançadas podem e devem ser feitas em qualquer unidade de atendimento de emergência. Por outro lado, o tratamento específico depende de centros de alta complexidade, pela necessidade de investigação com exames complementares como tomografia, angiografia digital ou angiotomografia, além de equipe de neurocirurgia com disponibilidade de material adequado e suporte de Unidade de Terapia Intensiva (CAPLAN, 2009; STEHBENS,1972). 


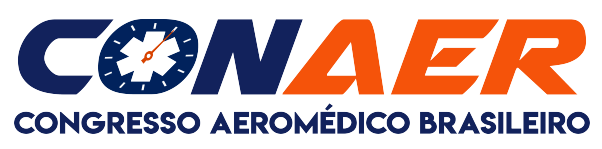

Compreende-se dessa forma que o transporte desses pacientes aos centros especializados deve ser feita de maneira rápida e segura de modo a possibilitar que o tratamento seja instituído o mais precocemente possível. Um dos transportes mais eficientes para este fim é o aeromédico com asa fixa, porém, o paciente poderá ser exposto a determinadas variáveis que podem agravar seu quadro clínico.

No transporte aeromédico com asa fixa, os ocupantes da aeronave serão submetidos a forças acelerativas em virtude das alterações rápidas na direção e na velocidade dos movimentos. Estas forças podem ser divididas em aceleração e desaceleração lineares - ocorrem na decolagem e no pouso - e aceleração centrífuga - ocorre durante curvas/mudanças de direção (HALL, 2011).

As forças de aceleração e desaceleração lineares dependem da quantidade de potência dos motores da aeronave durante a decolagem e, na aterrisagem, da velocidade de toque no solo e utilização de sistemas de frenagem. Portanto, quanto menor for a pista, mais potência será necessária para atingir a velocidade de início de voo e maior será a necessidade da utilização de sistemas de frenagem durante o pouso, e consequentemente, maiores serão as forças acelerativas lineares.

A força de aceleração centrífuga é determinada pela fórmula $f=m v^{2} / r$, ( $f$ : força de aceleração centrífuga, $m$ : massa do objeto, $v$ : velocidade do objeto; $r$ : raio da curva). Analisando esta fórmula, concluí-se que ela é diretamente proporcional ao quadrado da velocidade e inversamente ao raio da curva (DAVIS, 2008).

Estas forças são medidas através da escala de força $G$, na qual a força exercida apenas pela gravidade sobre um objeto que não está sob ação de outras forças acelerativas é igual a $+1 \mathrm{G}$. O principal efeito delas é sobre o sistema circulatório, devido ao deslocamento do sangue para as porções mais distantes de onde o movimento teve início. Este efeito promove acúmulo de sangue (hiperemia) nas regiões distais e carência (oliguemia) nas porções proximais (HALL, 2011).

Dentre os ocupantes da aeronave, os pacientes com AVCh possuem particularidades em relação às respostas a estas forças devido às alterações de pressão intracraniana (PIC) em voo. Em virtude da posição deitada do paciente, ele estará sujeito principalmente às forças acelerativas lineares e, se estiver com a cabeça voltada para a região anterior da aeronave, irá ocorrer diminuição transitória da pressão de perfusão cerebral (PPC) e da PIC durante a decolagem e o inverso durante a aterrisagem. Por outro lado, o oposto ocorrerá se estiver com a cabeça 
voltada para a região posterior (cauda da aeronave). Assim, o aumento da PPC e da PIC poderão ocasionar aumento do sangramento intracraniano (GUERRIERO, 2021).

Infelizmente, a literatura a respeito do assunto é escassa e não foram encontrados estudos ou publicações, nem tampouco relatos de efeitos deletérios em pacientes acometidos por AVCh transportados por aeronaves de asa fixa. Apesar desta escassez, pesquisas qualificam o cuidado no posicionamento do paciente na aeronave baseadas nos mecanismos fisiopatológicos, elevação da cabeceira do leito, monitoramento contínuo do paciente, preferência pelo uso de pista longa, redução de manobras bruscas em voo, aceleração e desaceleração suaves da aeronave. O somatório destas medidas permitirá o transporte de forma segura para centros especializados de tratamento do AVCh.

\section{CONSIDERAÇÕES FINAIS}

As hemorragias intracranianas são condições graves e que necessitam de investigação e tratamento em centros com estrutura adequada. Para isso, algumas vezes os pacientes precisam ser transportados por aeronaves de asa fixa.

Nesta modalidade de transporte os pacientes são submetidos a forças acelerativas lineares e centrífugas em maior ou menor grau na dependência da posição em que o paciente se encontra e da necessidade do uso de maior ou menor força de aceleração na decolagem, durante manobras e aterrisagem da aeronave. Como estes pacientes habitualmente apresentam aumento da PIC e a principal causa do sangramento está relacionada ao aumento da pressão arterial, devemos adotar medidas para minimizar tais efeitos.

Portanto, a utilização de aeronaves de asa fixa para o transporte destes pacientes parece ser segura desde que sejam utilizadas pistas longas, com menor necessidade de fortes acelerações para a decolagem ou desacelerações bruscas para o pouso, além de evitar manobras repentinas durante o voo.

A revisão das bases de dados epidemiológicos forneceu dados a respeito da doença em estudo, e a pesquisa em bases de dados com os termos relacionados anteriormente nos mostrou a carência de discussões mais precisas no transporte aeromédico do paciente com AVCh em aviões. 


\section{CONAER \\ CONGRESSO AEROMÉDICO BRASILEIRO}

\section{REFERÊNCIAS}

CAPLAN LR. Basic pathology, anatomy and pathophysiology of stroke. Caplan's stroke - a clinical approach. Ed 4. Editora Saunders Elsevier, 2009.

CAPLAN LR. Intracerebral hemorrhage revisited. Neurology 1988; 38: 624-627.

CAPLAN LR. Subarachnoid hemorrhage, aneurysms, and vascular malformations. Caplan's stroke - a clinical approach. Ed 4. Editora Saunders Elsevier, 2009.

COLE F, Yates P. Intracerebral microaneurysms and small cerebrovascular lesions. Brain 1967; 90:759-768.

DAVIS, JR et al. Human response to acceleration. Fundamentals of aerospace medicine. Ed 4. Editora Lippincott Williams \& Wilkins. 2008.

GUERRIERO, MP. Transporte e resgate aeromédico em patologias neurológicas.In: SUEOKA,JS. FREIXO, JAA;e TAVERNA M. Transporte e Resgate Aeromédico. Guanabara Koogan. Rio de Janeiro, 2021.

HALL JE. Efeitos das forças de aceleração sobre o corpo em fisiologia aeroespacial. Guyton \& Hall - Tratado de fisiologia médica. Ed. 12. Editora Elsevier. 2011.

HERBSTEIN D, Schaumberg H. Hypertensive intracerebral hematoma: An investigation of the initial hemorrhage and rebleeding using $\mathrm{Cr} 51$ labeled erythrocytes. Arch Neurol 1974;30: 412-414.

Indicadores de Dados Básicos - Brasil - 2012 Brasil. Disponível em http:// tabnet.datasus.gov.br/cgi/idb2012/matriz.htm acessado em 12 março 2021.

NAEMT - National Association of Emergency Medical Technicians. Pre Hospital Trauma Life Support. PHTLS: atendimento pré-hospitalar ao traumatizado. 8a ed. Burlington: Jones \& Bartlett Learning, 2017.

PHILLIPS, M. et al. Helicopter transport effectiveness of patients for primary percutaneous coronary intervention. Air Med J., v. 32, n. 3, p. 144-152, 2013.

SILBERGLEIT, R. et al. Cost-effectiveness of helicopter transport of stroke patients for thrombolysis. Acad Emerg Med, v. 10, n. 9, p. 966-972, 2003.

STEHBENS WE. Pathology of the Cerebral Blood Vessels. Editora Mosby Elsevier, 1972. 